\title{
Investigation of radio sources from the interplanetary scintillation Pushchino Survey
}

\section{Optical identification $\star$}

\author{
A. I. Kopylov ${ }^{1,3}$, V. S. Artyukh ${ }^{2,4}$, and F. G. Kopylova ${ }^{1,3}$ \\ 1 Special Astrophysical Observatory of RAS, Nizhnij Arkhyz, Karachaevo-Cherkesia, 369167, Russia \\ 2 Pushchino Radio Astronomy Observatory of the Lebedev Physical Institute of RAS, Pushchino, Moscow region, \\ 142290, Russia \\ 3 Isaac Newton Institute of Chile, SAO Branch, Russia \\ ${ }^{4}$ Isaac Newton Institute of Chile, Pushchino Branch, Russia
}

Received 15 January 2004 / Accepted 25 February 2004

\begin{abstract}
We present the results of optical identification of 248 interplanetary scintillating (IPS) radio sources from the Pushchino Survey (PS) in the area of $0.11 \mathrm{sr}$ with the center at $\alpha=10^{\mathrm{h}} 28^{\mathrm{m}}, \delta=+41^{\circ}$. All 260 counterparts of IPS radio sources from the 7C and FIRST catalogues, which had been found in Paper I, were considered. We used USNO- $B 1.0$ catalogue (limiting magnitude $m_{R} \approx 21$ ) for the optical identification and searched the literature to find optical data for fainter objects. Photometric and spectroscopic observations were conducted on the $1 \mathrm{~m}$ and $6 \mathrm{~m}$ telescopes of SAO RAS. Optical magnitudes or deep limits for 22 objects and redshifts for 26 ones have been obtained. In total, we collected optical data for 116 (68 with redshift) counterparts of the PS radio sources. For the subsample of the PS quasars (41 objects) the redshift distribution was compared to those of several other samples of quasars (BRL, 3CRR, MQS, B3-VLA, 7CRS I-III and PKS 0.25 Jy) which have complete or nearly complete redshift information.
\end{abstract}

Key words. catalogs - radio continuum: galaxies - galaxies: quasars: general - galaxies: photometry - surveys

\section{Introduction}

The Pushchino Survey (PS) of interplanetary scintillating (IPS) radio sources consists of 414 sources detected at $102 \mathrm{MHz}$ in the two regions of the total area of $0.144 \mathrm{sr}$ with the centers at $\alpha=06^{\mathrm{h}} 28^{\mathrm{m}}, \delta=45^{\circ}\left(125\right.$ IPS sources) and $\alpha=10^{\mathrm{h}} 28^{\mathrm{m}}, \delta=$ $41^{\circ}$ (289 IPS sources) (Artyukh \& Tyul'bashev 1996a; Artyukh et al. 1998). These regions correspond to the first two regions of the 7C survey conducted at $151 \mathrm{MHz}$ (McGilchrist et al. 1990). The survey was made with the Large Phased Array (LPA) of the Pushchino Radio Asronomy Observatory (PRAO). The sensitivity of the observations was $\sim 0.1 \mathrm{Jy}$. The resolution of the IPS method is $\sim 0$ ' 1 . A comparison with the catalogue of bright IPS sources (Purvis et al. 1987) showed that about $75 \%$ of the PS sources are newly detected as objects what include at least one sufficiently bright component with a size of $<1^{\prime \prime}$. The PS survey is complete for IPS sources with flux densities $>0.3 \mathrm{Jy}$. According to Artyukh \& Tyul'bashev (1996b) at this flux

Send offprint requests to: A. I. Kopylov, e-mail: akop@sao.ru

* Table 2 is only available in electronic form at the CDS via anonymous ftp to cdsarc.u-strasbg. fr $(130.79 .128 .5)$ or via http://cdsweb.u-strasbg.fr/cgi-bin/qcat?]/A+A/421/455 density the theoretically expected fraction of lost sources is $\sim 1 \%$, and the number of false sources $\sim 1$. It should be noted that the majority of the IPS sources from the PS have steep radio spectra $(\alpha>0.5)$ and a high degree of compactness $(\langle R\rangle=0.36)$ (Tyul'bashev 1997). The compactness $\mathrm{R}$ of a radio source is defined as the fraction of the total flux density of the radio source contained in a compact scintillating component. For calculation of the flux density of the scintillating component from the measured rms fluctuations it was assumed that the angular size of the (single) scintillating component is equal to 0.' 1 (Artyukh \& Tyul'bashev 1996a).

From several lines of indirect argumentation it was supposed (Tyul'bashev 1997) that the majority of faint scintillating radio sources should be quasars. To verify this supposition, optical identification and spectroscopic observations are needed.

As a first step we have improved the accuracy of positions for IPS sources in the region $\alpha=10^{\mathrm{h}} 28^{\mathrm{m}}, \delta=41^{\circ}$ by cross-identification with the 7C and FIRST radio catalogues. In Paper I (Artyukh et al. 2003) we have described the radio identification of IPS radio sources. In the second paper we present the results of optical identifications and our redshift measurements that were performed in 1999-2003. 
In Sect. 2 we describe optical identification of PS radio sources with POSS-I and POSS-II. In Sect. 3 the results of our photometric and spectroscopic observations are described. In Sect. 4 photometric and redshift data for 116 objects are presented, including the ones found in the literature. Redshift distributions for quasars from the PS and other surveys are compared in Sect. 5, and in Sect. 6 we give our conclusions.

\section{Optical identifications with POSS-I and POSS-II}

To identify the PS radio sources we used the USNO-B1.0 catalogue (Monet et al. 2003). It contains coordinates, magnitudes ( $B 1$ and $R 1$ for POSS-I plates, and $B 2, R 2$ and $I$ for POSS-II plates of the Palomar Observatory Sky Surveys) and classification (stellaricity) for objects brighter than $R \approx 21.0$. We took the nearest optical object to the radio position (the centroid for multicomponent radio sources) as the identification. For single component radio sources a maximum offset of $3^{\prime \prime}$ was adopted. The highest identification rate was found for red POSS-II plates. We adopted the magnitude system for these plates $(R 2)$ as the basic one.

Comparison with CCD measurements in the $R$ band for 16 quasars and 7 radiogalaxies in the range $m_{R}=17.2-20.5$ showed that the zero-points of the $R 2$ and CCD magnitude systems coincide within $0.1 \mathrm{mag}$. The rms difference was about $0.5 \mathrm{mag}$. Taking into account contributions of the errors of CCD magnitudes (0.1-0.2) and possible variability of objects $(\sim 0.3)$ we estimated a typical error for $R 2$ magnitudes of $\approx 0.4$. We found a high completeness of object detection in the USNO- $B 1.0$ catalogue down to $R \approx 20.5$. At fainter magnitudes a loss of objects begins.

\section{Observations}

We began the program of optical identifications of PS objects on telescopes of the SAO RAS in 1999. Our main aims were the spectroscopy of quasar candidates which had been confidently identified on the POSS-I, II and the search for fainter quasars $(R \sim 21)$ among the objects barely visible at the limit of POSS-II plates. Also a small subsample of most compact (compactness $R>0.5)$ and faint $\left(S_{m}<0.5 \mathrm{Jy}\right.$, that corresponds to $\left.S_{151}<1.0 \mathrm{Jy}\right)$ scintillating radio sources has been specially investigated. For these objects we conducted deep $\left(R_{\lim } \approx 23\right)$ photometric observations with the $1 \mathrm{~m}$ telescope and/or spectroscopic observations with the $6 \mathrm{~m}$ telescope.

\subsection{Photometric observations}

Photometric observations were conducted with several CCD detectors with the $1 \mathrm{~m}$ and $6 \mathrm{~m}$ telescopes. In 1999 the CCD photometer on the $1 \mathrm{~m}$ telescope had a ISD015A chip of $520 \times 580$ pixels, each $18 \times 24 \mu \mathrm{m}$, which corresponds to an angular size $0.276^{\prime \prime} \times 0.368^{\prime \prime}$. At the $6 \mathrm{~m}$ telescope we used a ISD017A chip of $1040 \times 1160$ pixels. In $2 \times 2$ binning mode the pixel size was $0.274^{\prime \prime}$. Observations were carried out with standard Johnson-Kron-Cousins filters $\left(B, V, R_{\mathrm{c}}, I_{\mathrm{c}}\right)$. Equatorial photometric standard stars from Landolt (1992) were used to determine transformation equations from our instrumental system into the standard one. To control extinction we observed the secondary standard stars in the fields of BL Lac objects MS0950.9+4929, MS1207.9+3945 and H1426+428 (Smith et al. 1991) at nearly the same zenith distance as our program objects.

In 2003 the $2048 \times 2052$ EEV CCD $42-40$ with a pixel size of $0.414^{\prime \prime}$ in $2 \times 2$ binning mode was employed at the $1 \mathrm{~m}$ telescope. With this CCD detector observations were carried out with a standard Kron-Cousins $R_{\mathrm{c}}$ filter. For typical integration times of $900-1800 \mathrm{~s}$ and seeing of $1.5^{\prime \prime}-2.5^{\prime \prime}$ the limiting magnitude was 22.5-23.5.

All objects, with the exception of PS090a, were observed in good or fair photometric conditions. The observational data were reduced using the ESO MIDAS package. The precision of photometry was better than $0.1-0.2$ mag for objects brighter than 21-22.5, and about $0.3 \mathrm{mag}$ for fainter ones. The results of our photometric measurements are presented in Table 1, which has the following contents: Col. 1 - name of the PS radio source; Col. 2 - date of observation; Col. 3 - telescope; Cols. 4-7 - magnitudes in $B, V, R_{\mathrm{c}}, I_{\mathrm{c}}$ bands; Col. 8 - type of object (Q - quasar, G - galaxy, EF - empty field), stellar objects without spectroscopic confirmation are marked as Q? and seemingly extended objects but near the limit of the CCD image as G?

\subsection{Spectroscopic observations}

Spectroscopic observations were conducted with the $6 \mathrm{~m}$ telescope. In 1999 we used the SP-124 spectrograph with the $1024 \times 1024$ PMCCD detector at the Nasmith-1 focus, in 2000 the UAGS spectrograph with the same detector at the prime focus. With these spectrographs redshifts for three quasars (PS123a, PS134a and PS266u) of 18.5-19.5 mag were measured. One of them (PS266u) was reobserved in 2003 and its redshift was confirmed. For PS134a our redshift exactly coinsides with the one given in Wold et al. (2000).

Since 2002 the 5 times more effective imagespectrograph SCORPIO (Afanas'ev \& Moiseev 2000; http://www . sao.ru/hq/moisav/scorpio/scorpio.html was used. With TK1024 CCD detector the quantum efficiency for this spectrograph is $30 \%$. The spectral range is 3700-9500 $\AA$ with a dispersion of about $6.5 \AA /$ pixel.

With the SCORPIO in 2002 and 2003 we measured new redshifts for 5 radiogalaxies and 16 quasars. For another two quasars (PS013a and PS044a) we confirmed redshifts from the literature.

All redshifts from our measurements are given in Table 2. A detailed description of our spectroscopic observations and measurements will be presented in a separate paper.

\section{Results}

85 out of 260 PS radio sources (33\%) were identified with objects from the USNO-B1.0 catalogue down to $R \lesssim 20.8$. A literature search showed that redshifts were available for 30 of them (21 quasars, 1 BL Lac object and 8 radiogalaxies). We also searched the literature to find optical data for fainter 
Table 1. Results of photometric observations.

\begin{tabular}{lccccccc}
\hline \hline PS & Date & Tel. & $B$ & $V$ & $R_{\mathrm{c}}$ & $I_{\mathrm{c}}$ & Type \\
\hline $005 \mathrm{a}$ & 03.02 .28 & $1 \mathrm{~m}$ & - & - & 21.2 & - & $\mathrm{G}$ \\
$011 \mathrm{u}$ & 99.02 .17 & $6 \mathrm{~m}$ & 21.2 & 21.0 & 20.4 & 20.1 & $\mathrm{Q}$ \\
$012 \mathrm{u}$ & 03.03 .05 & $1 \mathrm{~m}$ & - & - & 22.8 & - & $\mathrm{G} ?$ \\
$030 \mathrm{u}$ & 03.03 .05 & $1 \mathrm{~m}$ & - & - & 23.0 & - & $\mathrm{Q} ?$ \\
$049 \mathrm{a}$ & 03.02 .28 & $1 \mathrm{~m}$ & - & - & $>22.5$ & - & $\mathrm{EF}$ \\
$054 \mathrm{a}$ & 03.03 .04 & $1 \mathrm{~m}$ & - & - & 20.5 & - & $\mathrm{G}$ \\
$055 \mathrm{a}$ & 03.03 .04 & $1 \mathrm{~m}$ & - & - & 23.3 & - & $\mathrm{G} ?$ \\
$081 \mathrm{a}$ & 99.02 .17 & $6 \mathrm{~m}$ & 23.3 & 21.8 & 20.8 & 19.7 & $\mathrm{G}$ \\
$090 \mathrm{a} a$ & 03.03 .04 & $1 \mathrm{~m}$ & - & - & $23.0:$ & - & $\mathrm{G} ?$ \\
$109 \mathrm{a}{ }^{b}$ & 99.04 .17 & $1 \mathrm{~m}$ & - & - & $20.7:$ & - & $\mathrm{G}$ \\
$116 \mathrm{a}$ & 03.03 .05 & $1 \mathrm{~m}$ & - & - & 22.3 & - & $\mathrm{G}$ \\
$134 \mathrm{a}$ & 99.01 .18 & $1 \mathrm{~m}$ & 19.0 & - & 18.5 & - & $\mathrm{Q}$ \\
$149 \mathrm{c}$ & 03.03 .05 & $1 \mathrm{~m}$ & - & - & 21.9 & - & $\mathrm{G}$ \\
$169 \mathrm{a}$ & 03.03 .05 & $1 \mathrm{~m}$ & - & - & $>23.0$ & - & $\mathrm{EF}$ \\
$236 \mathrm{a}$ & 03.02 .28 & $1 \mathrm{~m}$ & - & - & $>22.5$ & - & $\mathrm{EF}$ \\
$266 \mathrm{u}$ & 99.04 .17 & $1 \mathrm{~m}$ & 20.2 & - & 19.6 & - & $\mathrm{Q}$ \\
$267 \mathrm{~b}$ & 03.02 .28 & $1 \mathrm{~m}$ & - & - & 19.8 & - & $\mathrm{Q}$ \\
$267 \mathrm{~d}$ & 03.03 .01 & $6 \mathrm{~m}$ & - & - & 22.3 & - & $\mathrm{G}$ \\
$274 \mathrm{u}$ & 99.05 .14 & $6 \mathrm{~m}$ & 22.4 & 21.9 & 21.3 & 20.5 & $\mathrm{Q} ?$ \\
$279 \mathrm{~b}$ & 99.04 .17 & $1 \mathrm{~m}$ & $>22.5$ & 20.9 & 20.1 & 18.9 & $\mathrm{G}$ \\
$284 \mathrm{~b}$ & 03.03 .01 & $1 \mathrm{~m}$ & - & - & 22.7 & - & $\mathrm{G} ?$ \\
$288 \mathrm{~b}$ & 99.04 .17 & $1 \mathrm{~m}$ & $21.4:$ & 20.0 & 19.0 & 18.3 & $\mathrm{G}$ \\
\hline
\end{tabular}

${ }^{a}$ Observed through cirrus, magnitude is uncertain. ${ }^{b}$ Blended with nearby galaxy, magnitude is uncertain.

objects. We found redshifts for 2 quasars and 13 radiogalaxies, mainly from the 6CE sample (Rawlings et al. 2001).

In total, optical data have been collected for 116 PS objects. They are presented in Table 2. Column 1 - name of the PS object according to the convention adopted in Paper I; Cols. 2-4 optical coordinates (or radio coordinates for empty fields) and reference; Col. 5 - flux density of scintillating radio source at $102 \mathrm{MHz}$; Col. 6 - flux density at $151 \mathrm{MHz}$; Cols. 7, 8-magnitude in $R$-band and original band(s) of observation (see notes for the table); Cols. 9, 10 - redshift and type of object (as in Table 1); Cols. 11, 12 - references for magnitude and redshift, respectively.

Figure 1 shows the number vs. magnitude distribution for 116 PS objects from Table 2. Most of the 15 quasar candidates without redshift measurement have magnitudes in the range $m_{R}=19-20.5$. The combined distribution on $R$ magnitude for quasars and quasar candidates peaks at 19.5-20. This means that among 144 optically unidentified objects, which have only a magnitude limit from the POSS-II $\left(m_{R} \gtrsim 21\right)$, the fraction of quasars is probably not high. An order of magnitude evaluation of quasar fraction of $\lesssim 15 \%$ could be made using the number of quasars and quasar candidates (5 objects) among 29 optically identified objects with $m_{R} \geqslant 20.8$ in Table 2 . All these objects were not detected in $R 2$ band in the USNO- $B 1.0$ catalogue and only one quasar candidate, PS028a, was detected in B2-band.

The distribution of redshift vs. magnitude for the PS quasars and radiogalaxies is shown in Fig. 2. For comparison we marked by two dotted lines the band which should be populated by giant elliptical galaxies according to the evolutionary population synthesis model of Poggianti (1997). Most

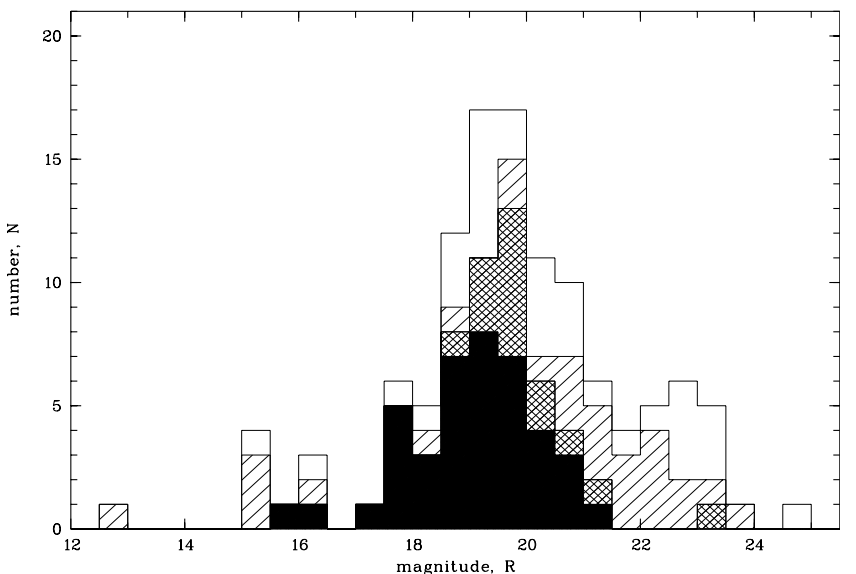

Fig. 1. Number vs. magnitude distribution for 116 PS radio sources from Table 2: 41 quasars with $z$ (filled area of histogram), 15 quasar candidates without $z$ (double-hashed area), 27 radiogalaxies with $z$ (single-hashed area), and 33 radiogalaxies without $z$ (blank area), including three unidentified objects shown at their CCD limits $(R>$ 22.5).

of the PS radiogalaxies fall between these lines, but the completeness of the subsample of radiogalaxies with measured redshifts is very low.

\section{Comparison of quasar samples}

In Fig. 3 we compared redshift distributions for several complete samples of quasars: equatorial BRL (Best et al. 1999), 3CRR (Willott et al. 1998; Willott et al. 1999), MQS (Kapahi et al. 1998), B3-VLA (Vigotti et al. 1997), 7CRS I-III 


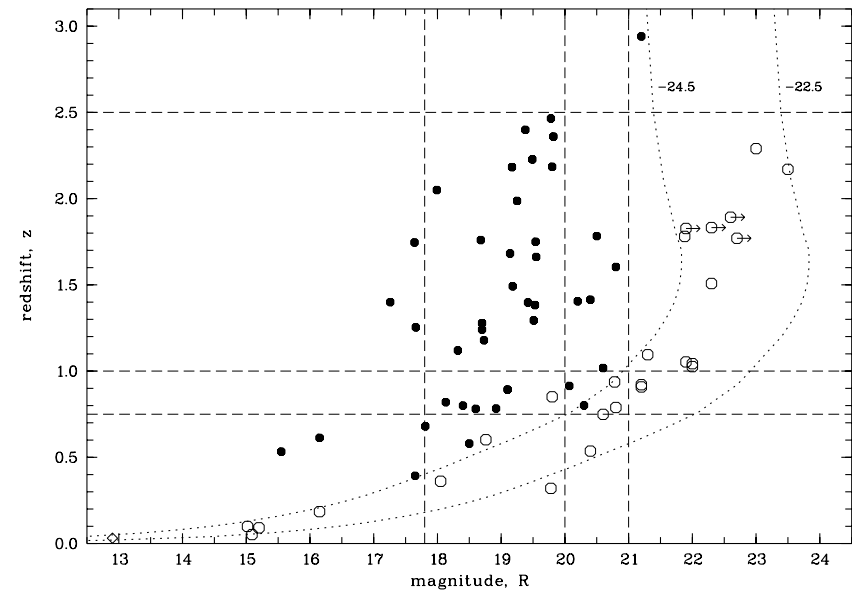

Fig. 2. Redshift vs. magnitude distribution of the PS radio sources. Quasars are shown by filled circles, radiogalaxies - by open circles, and one BL Lac object - by losange. The model of evolution of elliptical galaxies (Poggianti 1997) for cosmological parameters $H_{0}=50 \mathrm{~km} \mathrm{~s}^{-1} / \mathrm{Mpc}$ and $q_{0}=0.225$ is shown by dotted lines for two values of absolute magnitude. Vertical dashed lines mark magnitude limits for the FBQS sample $(R=17.8)$, POSS-I $(R=20)$ and POSS-II $(R=21)$. Two bottom horizontal dashed lines correspond to the approximate redshift limits for radiogalaxies visible on POSS-I and POSS-II. The effective "redshift cut-off" for the present sample of PS objects is shown by the upper horizontal dashed line.

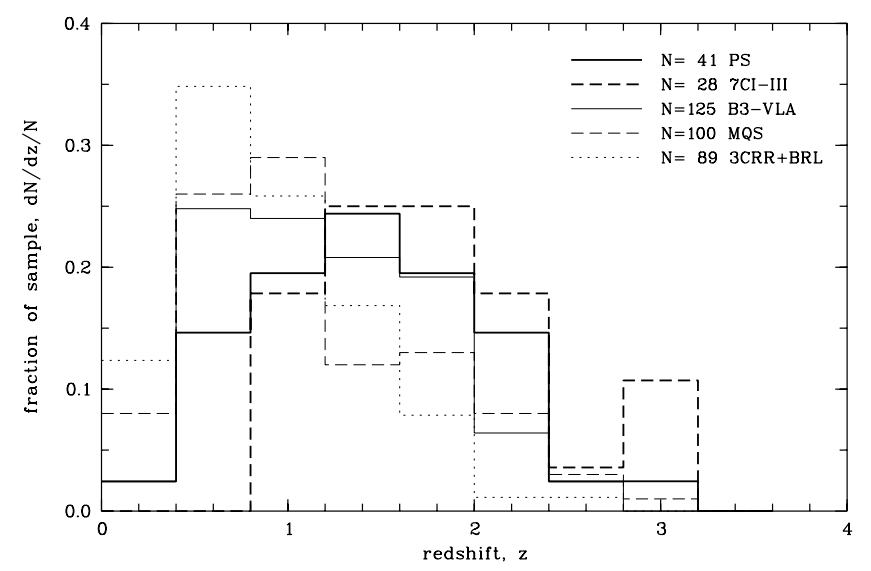

Fig. 3. Redshift distribution for quasars from the PS sample and four other samples of radiosources with complete or nearly complete redshift data. In all samples a proportion of flat spectrum quasars $(\alpha<$ $0.5)$ is approximately the same $(17 \pm 4 \%)$.

(Willott et al. 1998; Lacy et al. 1999), PKS 0.25 Jy (flat spectrum) (Jackson et al. 2002). The main parameters for these samples are given in Table 3. In the last column of this table a median $z$ is given. From the BRL sample we have excluded two objects, 0834-196 and 1116-027 (3C255), which were classified as radiogalaxies according to the data from Stickel \& Kühr (1996) and Giraud (1990), respectively. The B3-VLA sample consists of quasars identified down to $m_{R} \approx 20$. For the PKS $0.25 \mathrm{Jy}$ sample the redshift completeness is about $72 \%$.

From Fig. 3 we have found that by their redshift distribution the PS quasars are most similar to B3-VLA and 7CRS quasars. In fact, histograms for B3-VLA and 7CRS quasars bracket the

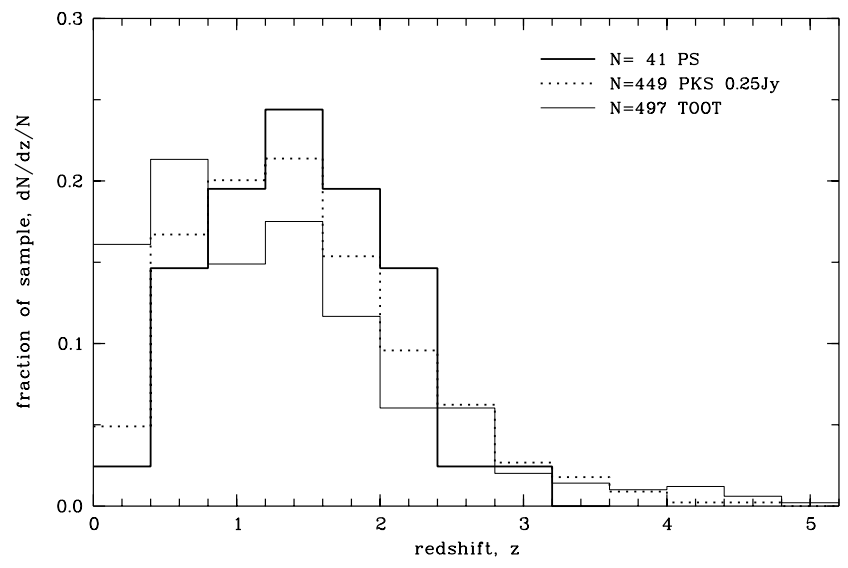

Fig. 4. Redshift distribution for quasars from the PS sample (thick solid line) and flat spectrum quasars from the PKS $0.25 \mathrm{Jy}$ sample (dotted line). For comparison the preliminary data from Hill \& Rawlings (2003) for the complete sample of radio sources (TOOT) with limiting flux density $S_{151}>0.1 \mathrm{Jy}$ are shown (thin solid line).

one for PS in all redshift bins, but the fractions of B3-VLA are higher for $z<1.2$ and lower for $z>1.2$, and vise versa for 7CRS.

Comparison with the PKS flat spectrum quasars is presented in Fig. 4. Distributions of the PS and PKS quasars are not significantly different (at the level of 0.05 according to the Smirnov test). The preliminary redshift distribution from Hill \& Rawlings (2003) for the complete sample of radio sources (TOOT) with the limiting flux density $S_{151}>0.1 \mathrm{Jy}$ is also shown in Fig. 4.

\section{Conclusions}

We have conducted systematic investigation of a sample of faint scintillating radio sources detected in the Pushchino Survey. Our observations and search of the literature have provided optical identification (magnitude and/or redshift) for 113 out of 260 radio counterparts found in the 7C and FIRST catalogues for 248 scintillating PS radiosources. The remaining 147 counterparts $(57 \%)$ are unidentified in optics down to the limiting magnitude of the POSS-II $(R \approx 21, B \approx 22)$. For 3 of them we have obtained deeper CCD limits $(R>22.5-23)$.

Redshifts are available for 68 objects: 41 quasars, 1 BL Lac object and 26 radiogalaxies. For quasar candidates found so far the completeness of spectroscopy is about $75 \%$. We plan to complete the redshift survey of quasar candidates in 2004. It is expected that among the optically faint PS objects the vast majority are radiogalaxies. The fraction of quasars may nevertheless be as high as $10-15 \%$.

We have found that for quasars from the PS sample and other samples of comparable flux densities $\left(S_{151} \gtrsim 0.5 \mathrm{Jy}\right)$ the redshift distributions are similar and show an effective "redshift cut-off" at $z=2.5-3$.

Acknowledgements. We thank A. V. Moiseev, V. P. Mikhajlov and S. N. Dodonov for their support during the spectroscopic observation with the SCORPIO on the $6 \mathrm{~m}$ telescope and V. L. Afanas'ev for providing a software used for reduction of spectra and redshift measurements. The authors made use of the database CATS 
Table 3. Samples of quasars.

\begin{tabular}{lccrcccc}
\hline \hline Sample & $\begin{array}{c}v \\
(\mathrm{MHz})\end{array}$ & $\begin{array}{c}S_{\lim } \\
(\mathrm{Jy})\end{array}$ & $N$ & $z_{\min }$ & $z_{\max }$ & $\langle z\rangle$ & $z_{\text {med }}$ \\
\hline BRL & 408 & 5.0 & 47 & 0.158 & 2.594 & 0.88 & 0.72 \\
3CRR & 178 & 10.9 & 42 & 0.305 & 2.012 & 1.00 & 0.92 \\
BRL+3CRR & & & 89 & 0.158 & 2.594 & 0.94 & 0.85 \\
MQS & 408 & 0.95 & 100 & 0.132 & 2.914 & 1.13 & 0.94 \\
B3-VLA & 408 & $0.1-1.6$ & 125 & 0.096 & 2.753 & 1.24 & 1.16 \\
PS & 102 & $\sim 0.5$ & 41 & 0.393 & 2.941 & 1.42 & 1.40 \\
7CRS I-III & 151 & 0.5 & 28 & 0.832 & 2.982 & 1.73 & 1.64 \\
PKS 0.25 Jy & 2700 & $\approx 0.25$ & 449 & 0.158 & 4.464 & 1.46 & 1.35 \\
\hline
\end{tabular}

(Verkhodanov et al. 1997) of the Special Astrophysical Observatory and VizieR facilities at CDS (Ochsenbein et al. 2000). This work was supported by the Russian Foundation for Basic Research (project code 01-02-16191a).

\section{References}

Allington-Smith, J. R., Perryman, M. A. C., Longair, M. S., Gunn, J. E., \& Westphal, J. A. 1982, MNRAS, 201, 331

Artyukh, V. S., Kopylov, A. I., \& Kopylova, F. G. 2003, A\&A, 403, 555 (Paper I)

Artyukh, V. S., \& Tyul'bashev, S. A. 1996a, Astron. Rep., 40, 601

Artyukh, V. S., \& Tyul'bashev, S. A. 1996b, Astron. Rep., 40, 608

Artyukn, V. S., \& Tyul'bashev, S. A. 1998, Astron. Rep., 42, 576

Artyukh, V. S., Tyul'bashev, S. A., \& Isaev, E. A. 1998, Astron. Rep., 42,283

Best, P. N., Longair, M. S., \& Röettgering, J. H. A. 1997, MNRAS, 292, 758

Best, P. N., Röettgering, J. H. A., \& Lehnert, M. D. 1999, MNRAS, 310,223

Bicker, J., Fritze - v. Alvensleben, U., Moeller, C. S., \& Fricke, K. J. 2004, A\&A, 413, 37

Carballo, R., González-Serrano, J. I., Benn, C. R., Sánchez, S. F., \& Vigotti, M. 1999, MNRAS, 306, 137

Eales, S. A. 1985, MNRAS, 217, 167

Eales, S. A., \& Rawlings, S. 1996, ApJ, 460, 68

Eales, S., Rawlings, S., Law-Green, D., Cotter, G., \& Lacy, M. 1997, MNRAS, 291, 593

Fanti, C., Pozzi, F., Dallacasa, D., et al. 2001, A\&A, 369, 380

Gandhi, P., Crawford, C. S., Fabian, A. C., \& Johnstone, R. M. 2003, MNRAS, in press [arXiv: astro-ph/0310772]

Giraud, E. 1990, A\&A, 234, 20

Hill, G. J., \& Rawlings, S. 2003 [arXiv: astro-ph/0301441]

Inskip, K. J., Best, P. N., Longair, M. S., et al. 2003, MNRAS, 345,1365

Jackson, C. A., Wall, J. V., Shaver, P. A., et al. 2002, A\&A, 386, 97

Jarvis, M. J., Rawlings, S., Eales, S., et al. 2001, MNRAS, 326, 1585

Kapahi, V. K., Athreya, R. M., Subrahmanya, C. R., et al. 1998, ApJS, 118,327

Lacy, M., Rawlings, S., Hill, G. J., et al. 1999, MNRAS, 308, 1096

Landolt, A. U. 1992, AJ, 104, 340
Lavery, R. J., Owen, F. N., \& Henry, J. P. 1993, BAAS, 25, 1307

Law-Green, J. D. 1995, in Proc. of the XXVIIth Young European Radio Astronomers Conference, ed. D. A. Green and W. Steffen, 18

Lilly, S. J. 1989, ApJ, 340, 77

Machalski, J. 1998, A\&AS, 128, 153

Maxfield, L., Thompson, D., Djorgovski, S., Vigotti, M., \& Grueff, G. 1995, PASP, 107, 369

McCarthy, P. J. 1991, AJ, 102, 518

McGillchrist, M., Baldwin, J. E., Riley, J. M., et al. 1990, MNRAS, 246,110

Monet, D. G., Levine, S. E., Canzian, B., et al. 2003, AJ, 125, 984

Naundorf, C. E., Alexander, P., Riley, J. M., \& Eales, S. A. 1992, MNRAS, 258, 647

Ochsenbein, F., Bauer, P., \& Marcout, J. 2000, A\&AS, 143, 221

Poggianti, B. M. 1997, A\&AS, 122, 399

Rawlings, S., Eales, S., \& Lacy, M. 2001, MNRAS, 322, 523

Sandage, A. 1978, AJ, 83, 904

Smith, P. S., Jannuzi, B. T., \& Elston, R. 1991, ApJS, 77, 67

Spinrad, H., Marr, J., Aguilar, L., \& Djorgovski, S. 1985, PASP, 97, 932

Stickel, M., \& Kühr, H. 1996, A\&AS, 115, 11

Thompson, D., Djorgovski, S., Vigotti, M., \& Grueff, G. 1994, AJ, 108,828

Tyul'bashev, S. A. 1997, Astron. Rep., 41, 723

Verkhodanov, O. V., Trushkin, S. A., Andernach, H., \& Chernenkov, V. N. 1997, The CATS database to operate with astrophysical catalogs. In Astronomical Data Analysis Software and Systems VI, ed. G. Hunt, \& H. E. Payne, ASP Conf. Ser., 125, 322

Véron-Cetty, M.-P., \& Véron, P. 2001, A\&A, 374, 92

Vigotti, M., Vettolani, G., Merighi, R., Lahulla, J. F., \& Pedani, M. 1997, A\&AS, 123, 219

White, R. L., Becker, R. H., Helfand, D. J., \& Gregg, M. D. 1996, ApJ, 475,479

Willott, C. J., Rawlings, S., Blundell, K. M., \& Lacy, M. 1998, MNRAS, 300, 625

Willott, C. J., Rawlings, S., Blundell, K. M., \& Lacy, M. 1999, MNRAS, 309, 1017

Willott, C. J., Rawlings, S., Archibald, E. N., \& Dunlop, J. S. 2002, MNRAS, 331, 435

Wold, M., Lacy, M., Lilje, P. B., \& Serjeant, S. 2000, MNRAS, 316, 267 